\title{
Phonon-mediated superconductivity in compressed $\mathrm{NbH}_{4}$ compound
}

\author{
Artur P. Durajski ${ }^{a}$ \\ Institute of Physics, Czȩstochowa University of Technology, Ave. Armii Krajowej 19, 42-200 Czȩstochowa, Poland
}

Received 8 May 2014 / Received in final form 21 July 2014

Published online 16 September 2014

(C) The Author(s) 2014. This article is published with open access at Springerlink.com

\begin{abstract}
There has been much interest in the superconducting properties of metal hydrides for the last few years. The hydrogen-rich compounds are regarded as a way of hydrogen metallization at lower pressures than that required for pure hydrogen. This paper deals with the thermodynamic properties of the superconducting state in niobium hydride $\left(\mathrm{NbH}_{4}\right)$ at $300 \mathrm{GPa}$. All theoretical calculations have been conducted within the framework of the strong-coupling Eliashberg theory. It has been stated that the critical temperature $\left(T_{C}\right)$ is equal to $49.57 \mathrm{~K}$ when the Coulomb pseudopotential takes the commonly accepted value of 0.1 . In the considered case, the ratio of the energy gap to the critical temperature $\left(R_{\Delta} \equiv 2 \Delta(0) / k_{B} T_{C}\right)$, the ratio of the specific heat jump to the heat of the normal state $\left(R_{C} \equiv \Delta C\left(T_{C}\right) / C^{N}\left(T_{C}\right)\right)$ and the ratio connected with the thermodynamic critical field $\left(R_{H} \equiv T_{C} C^{N}\left(T_{C}\right) / H_{C}^{2}(0)\right)$ are equal to $3.91,2.12$ and 0.154 , respectively. Above results differ significantly from the values predicted by the weak-coupling Bardeen-Cooper-Schrieffer theory, which omits a very important role of the strong-coupling and phonon retardation effects.
\end{abstract}

\section{Introduction}

Since Ashcroft suggested the possibility of the existence of superconducting state in a metallic hydrogen [1] and in the dense group IVa hydrides [2] there has been great interest in compressed hydrogen-rich compounds [3-5]. These materials are promising candidates for high-temperature superconductors at lower pressures than pure hydrogen. Such a situation may occur owing to chemical precompression exerted by heavier atoms in compounds with a large hydrogen content [2].

For example, in the case of silane $\left(\mathrm{SiH}_{4}\right)$ independent experimental and theoretical works [6-8] have confirmed the metallization at pressures of the order of 50-60 GPa. Moreover, the transition from the normal to the superconducting state with critical temperature $\left(T_{C}\right)$ of $17 \mathrm{~K}$ has been observed at 96 and $120 \mathrm{GPa}$ [6].

On the other hand, in paper [9] the decomposition of silane has been observed at a higher pressure of $50 \mathrm{GPa}$ and the transition to the metallic phase has not been observed up to at least $130 \mathrm{GPa}$ [10]. As a result of the discussion, it was suggested that the previously measurements could relate the $\mathrm{PtH}$ compound that has been formed upon the decomposition of silane and the reaction of released hydrogen with platinum metal that is present in the diamond anvil cell [9]. These suppositions were confirmed by performing the first-principles calculations $[11,12]$. However, there are currently no reports on the experimental results.

\footnotetext{
${ }^{a}$ e-mail: adurajski@wip.pcz.pl
}

Other high-pressure hydrogenated materials have been investigated mainly theoretically so far. The results presented in the literature suggest the existence of the superconducting state at relatively high critical temperature exceeding the boiling point of liquid nitrogen. In particular, in the family of hydrogenated silicon: $\mathrm{Si}_{2} \mathrm{H}_{6}$ at $275 \mathrm{GPa}$ and $\mathrm{SiH}_{4}\left(\mathrm{H}_{2}\right)_{2}$ at $250 \mathrm{GPa}$, application of the Allen-Dynes modified McMillan equation gives the highest superconducting transition temperatures amounts $153 \mathrm{~K}$ and $107 \mathrm{~K}$, respectively $[13,14]$. In hydrogenated germane $\mathrm{GeH}_{4}\left(\mathrm{H}_{2}\right)_{2}$ at $250 \mathrm{GPa}, T_{C}$ is equals to $90 \mathrm{~K}[15]$ and hydrogenated gallane $\mathrm{GaH}_{3}$ at $120 \mathrm{GPa}$ is characterized by $T_{C}=102 \mathrm{~K}[16]\left(T_{C}\right.$ rises to $123 \mathrm{~K}$ when calculations are conducted in the framework of the Eliashberg formalism [17]).

One of the more interesting predictions is the existence of the superconducting state in $\mathrm{CaH}_{6}$ compound at critical temperature equal to $235 \mathrm{~K}$ or $243 \mathrm{~K}$, depending on the assumed method $[18,19]$. It should be noted that calcium holds the record for the highest superconducting transition temperature $\left(T_{C}=29 \mathrm{~K}\right)$ among all elements at high pressure $(p=220 \mathrm{GPa})$ [20].

Motivated by the recent experimental and theoretical progress in this area, we have carried out numerical calculation to explore in detail the thermodynamic properties of superconducting state in hydrogenated niobium [21]. Let us note that at the atmospheric pressure, niobium has the highest critical temperature of the elemental superconductors $(9.2 \mathrm{~K})$. The theoretical studies conducted for $\mathrm{NbH}, \mathrm{NbH}_{2}$ and $\mathrm{NbH}_{4}$ also suggest an existence of 
the superconducting state. However, at normal condition stable are only $\mathrm{NbH}$ and $\mathrm{NbH}_{2}$ with critical temperatures lower than pure niobium, in particular $\left[T_{C}\right]_{\mathrm{NbH}}=2.4 \mathrm{~K}$ and $\left[T_{C}\right]_{\mathrm{NbH}_{2}}=2.6 \mathrm{~K} . \mathrm{NbH}_{4}$ is stable solely at high pressure and can be a candidate for high-temperature superconductor with a preliminary estimate of $T_{C}$ around $47 \mathrm{~K}$ [21]. Therefore, we focused our attention only on this case.

Due to the high value of the electron-phonon coupling constant $(\lambda=0.82)$ predicted for $\mathrm{NbH}_{4}$ our studies have been conducted within the framework of the strongcoupling Eliashberg formalism [22]. It should be underlined that materials with strong electron-phonon coupling are poorly described by Bardeen-Cooper-Schrieffer (BCS) theory of superconductivity. In these strong-coupling materials, phonon retardation effects play a very important role [23]. A proper treatment of those effects was developed in Eliashberg theory which allows to reproduce the superconducting properties of a conventional superconductor within an experimental accuracy. This approach has been described in paper [24] and successfully used in the study of other hydrogen-rich compounds [17,19,25], and also in the study of materials for which is a possibility of comparison of the theoretical and experimental results (e.g. simple elements: $\mathrm{Ca}, \mathrm{S}, \mathrm{Li}$, and compounds: $\left.\mathrm{CaLi}_{2}, \mathrm{~K}_{3} \mathrm{C}_{60}, \mathrm{Rb}_{3} \mathrm{C}_{60}, \mathrm{MgB}_{2}\right)$ [26-31]. Let us note that our calculated results agree quantitatively with the available experimental data.

The central quantity in Eliashberg theory is the electron-phonon spectral function $\alpha^{2} F(\Omega)$ which can be determined from tunnelling experiments or calculated theoretically. In presented paper, all calculations were based on the $\alpha^{2} F(\Omega)$ function determined by using the planewave pseudopotential method within density functional perturbation theory in paper [21].

\section{Eliashberg formalism}

The superconducting properties of conventional superconductors (materials with phonon-mediated pairing) can be calculated within experimental accuracy by solving the Eliashberg equations. The Eliashberg equations, formulated on the imaginary-axis, can be written as $[22,32]$ :

$$
Z_{n}=1+\frac{1}{\omega_{n}} \frac{\pi}{\beta} \sum_{m=-M}^{M} \frac{\lambda\left(i \omega_{n}-i \omega_{m}\right)}{\sqrt{\omega_{m}^{2} Z_{m}^{2}+\phi_{m}^{2}}} \omega_{m} Z_{m}
$$

and

$$
\phi_{n}=\frac{\pi}{\beta} \sum_{m=-M}^{M} \frac{\lambda\left(i \omega_{n}-i \omega_{m}\right)-\mu^{*} \theta\left(\omega_{c}-\left|\omega_{m}\right|\right)}{\sqrt{\omega_{m}^{2} Z_{m}^{2}+\phi_{m}^{2}}} \phi_{m} .
$$

In equations (1) and (2), $Z_{n} \equiv Z\left(i \omega_{n}\right)$ is the electron mass renormalization parameter due to electron-phonon interaction, and $\phi_{n} \equiv \phi\left(i \omega_{n}\right)$ is the order parameter function. Symbol $\omega_{n} \equiv(\pi / \beta)(2 n-1)$, with $n=0, \pm 1, \pm 2, \ldots$ is the $n$th Matsubara frequency. $\beta$ denotes the inverse temperature $\beta \equiv\left(k_{B} T\right)^{-1}$, where $k_{B}$ is the Boltzmann
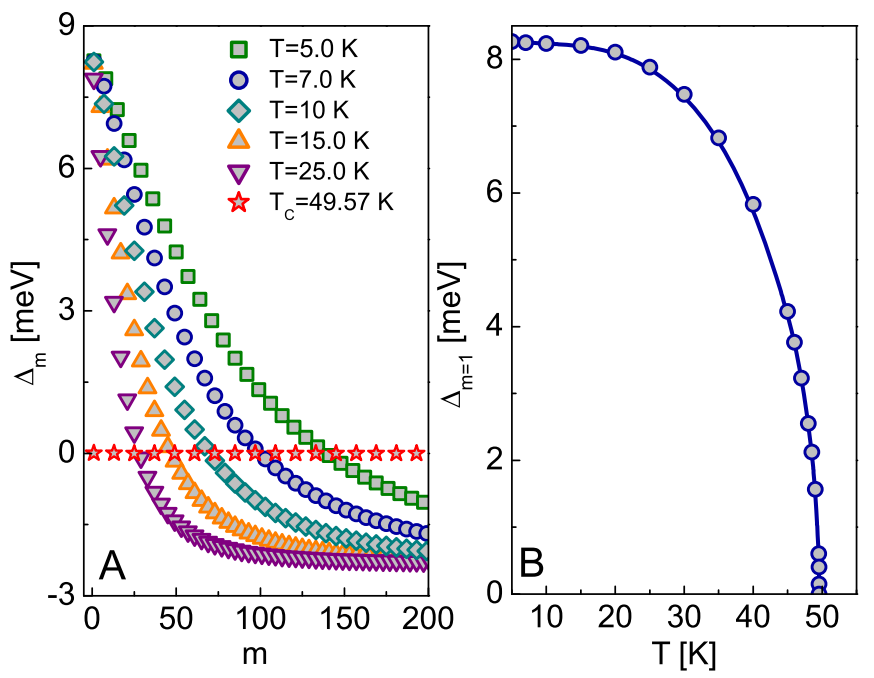

Fig. 1. (A) The order parameter on the imaginary axis for the selected values of the temperature. (B) The full dependency of the maximum value of the order parameter as a function of the temperature.

constant. The pairing electron-phonon interaction is contained in the kernel:

$$
\lambda(z) \equiv 2 \int_{0}^{\Omega_{\max }} d \Omega \frac{\Omega}{\Omega^{2}-z^{2}} \alpha^{2} F(\Omega),
$$

where the value of the maximum phonon frequency $\left(\Omega_{\max }\right)$ is equal to $275 \mathrm{meV}$. The effective Coulomb depairing interaction between the electrons is described by the dimensionless Coulomb pseudopotential $\mu^{\star}$. The quantity $\theta$ denotes the Heaviside function, and $\omega_{c}$ is a cut-off frequency, chosen as ten times the maximum phonon frequency: $\omega_{c}=10 \Omega_{\max }$.

The Eliashberg equations have been solved for 2201 Matsubara frequencies $(M=1100)$ with a typical choice of Coulomb pseudopotential $\mu^{\star}$ as 0.1 . In the considered case, the convergence of the solutions has been obtained from $T_{0}=5 \mathrm{~K}$.

\section{Results and discussion}

The dependency of the order parameter, defined as the ratio: $\Delta_{m} \equiv \phi_{m} / Z_{m}$, on the number of the Matsubara frequencies $m$ for the selected values of the temperature and the full dependency of $\Delta_{m=1}(T)$ have been presented in Figures $1 \mathrm{~A}$ and $1 \mathrm{~B}$, respectively. We can notice that the maximum value of the order parameter $\left(\Delta_{m=1}\right)$ is decreasing with the growth of the temperature. This property allows us to defined $T_{C}$ as the temperature at which the order parameter becomes zero. In the case of $\mathrm{NbH}_{4}$ at $300 \mathrm{GPa} T_{C}=49.57 \mathrm{~K}$. At this point it should be noted that the obtained result is different from the results provided by the analytical McMillan (McM) and Allen-Dynes (AD) equations $[33,34]$. In particular, $\left[T_{C}\right]_{\mathrm{McM}}=44.22 \mathrm{~K}$ and $\left[T_{C}\right]_{\mathrm{AD}}=46.77 \mathrm{~K}$. This means that the value of the 

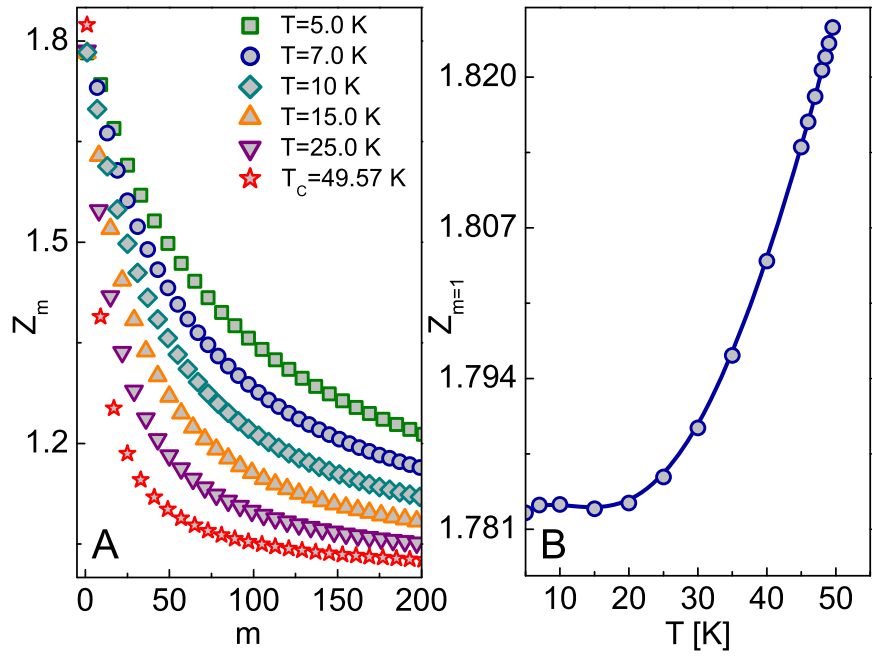

Fig. 2. (A) The wave function renormalization factor on the imaginary axis for the selected values of the temperature. (B) The full dependency of the maximum value of the wave renormalization factor as a function of the temperature.

critical temperature in a strict way can be determined using the Eliashberg equations. Recently, it has been proved for various conventional phonon-mediated superconductors in which the critical temperatures have been also determined experimentally [26,29].

In Figure 2, the form of the wave function renormalization factor on the imaginary axis, has been presented. Like in the case of the order parameter, for the increasing values of the Matsubara frequencies the values of $Z_{m}$ are decreasing. However, in comparison with the order parameter, the decrease is much slower (Fig. 2A). The dependence of the maximum value of the wave function renormalization factor on the temperature has been presented in Figure 2B. It can be seen, that together with the growth of temperature $\mathrm{Z}_{m=1}$ slightly increases and reaches maximum at $T_{C}$.

Thermodynamic properties of a superconductor can be calculated from the free energy difference between the superconducting and the normal state $\Delta F$. Based on the imaginary frequency axis solutions of the Eliashberg equations, the $\Delta F$ function has been calculated:

$$
\begin{aligned}
\frac{\Delta F}{\rho(0)}= & -\frac{2 \pi}{\beta} \sum_{n=1}^{M}\left(\sqrt{\omega_{n}^{2}+\Delta_{n}^{2}}-\left|\omega_{n}\right|\right) \\
& \times\left(Z_{n}^{S}-Z_{n}^{N} \frac{\left|\omega_{n}\right|}{\sqrt{\omega_{n}^{2}+\Delta_{n}^{2}}}\right),
\end{aligned}
$$

where $\rho(0)$ is the value of the electron density of states at the Fermi energy level. Symbols $Z_{n}^{S}$ and $Z_{n}^{N}$ denote the wave function renormalization factors for the superconducting and the normal state, respectively.

In the next steep, the thermodynamic critical field has been calculated from the free energy difference:

$$
\frac{H_{C}}{\sqrt{\rho(0)}}=\sqrt{-8 \pi[\Delta F / \rho(0)]} .
$$

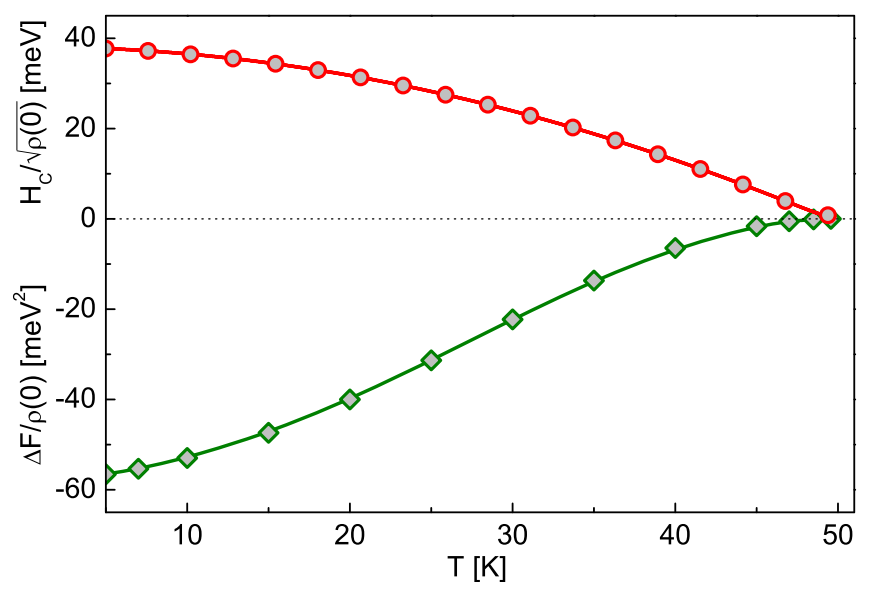

Fig. 3. The free energy difference $(\Delta F / \rho(0))$ and the thermodynamic critical field $\left(H_{C} / \sqrt{\rho(0)}\right)$ as a function of the temperature.

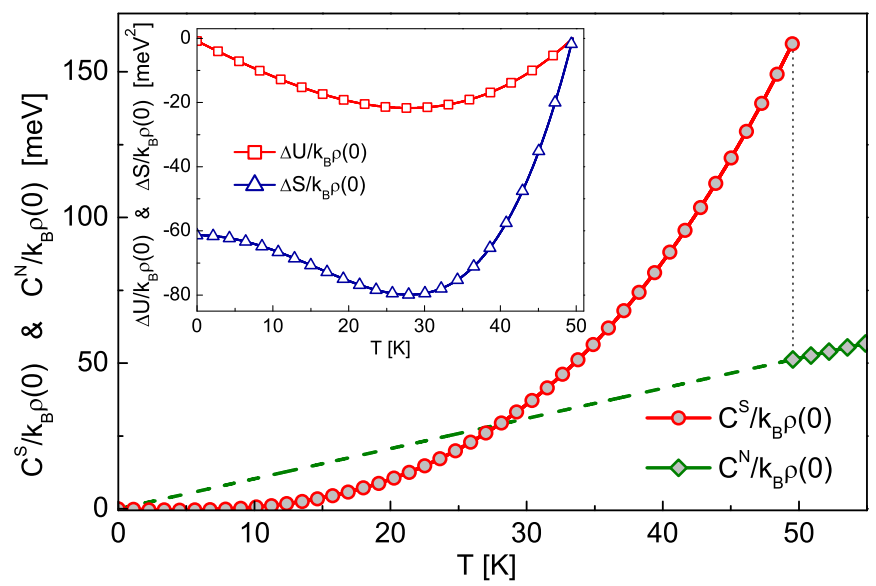

Fig. 4. The specific heat for the superconducting and the normal state as a function of the temperature. The inset shows the differences of entropy $(\Delta S)$ and internal energy $(\Delta U)$.

The temperature dependencies of $\Delta F / \rho(0)$ and $H_{C} / \sqrt{\rho(0)}$ have been shown in Figure 3 .

The difference in the specific heat between the superconducting and the normal state $\left(\Delta C=C^{S}-C^{N}\right)$ should be calculated using the expression:

$$
\frac{\Delta C(T)}{k_{B} \rho(0)}=-\frac{1}{\beta} \frac{d^{2}[\Delta F / \rho(0)]}{d\left(k_{B} T\right)^{2}} .
$$

The specific heat in the normal state can be calculated from: $C^{N}(T) / k_{B} \rho(0)=\gamma / \beta$, where the Sommerfeld constant is given by: $\gamma \equiv(2 / 3) \pi^{2}(1+\lambda)$. The dependencies of the specific heats on the temperature have been presented in Figure 4. Moreover, the inset of Figure 4 shows entropy $(\Delta S)$ and internal energy $(\Delta U)$ differences between the superconducting and the normal state calculated from the following equations [35]:

$$
\Delta S \equiv \int_{T_{\mathrm{C}}}^{T} \frac{C^{S}(T)-C^{N}(T)}{T} d T
$$



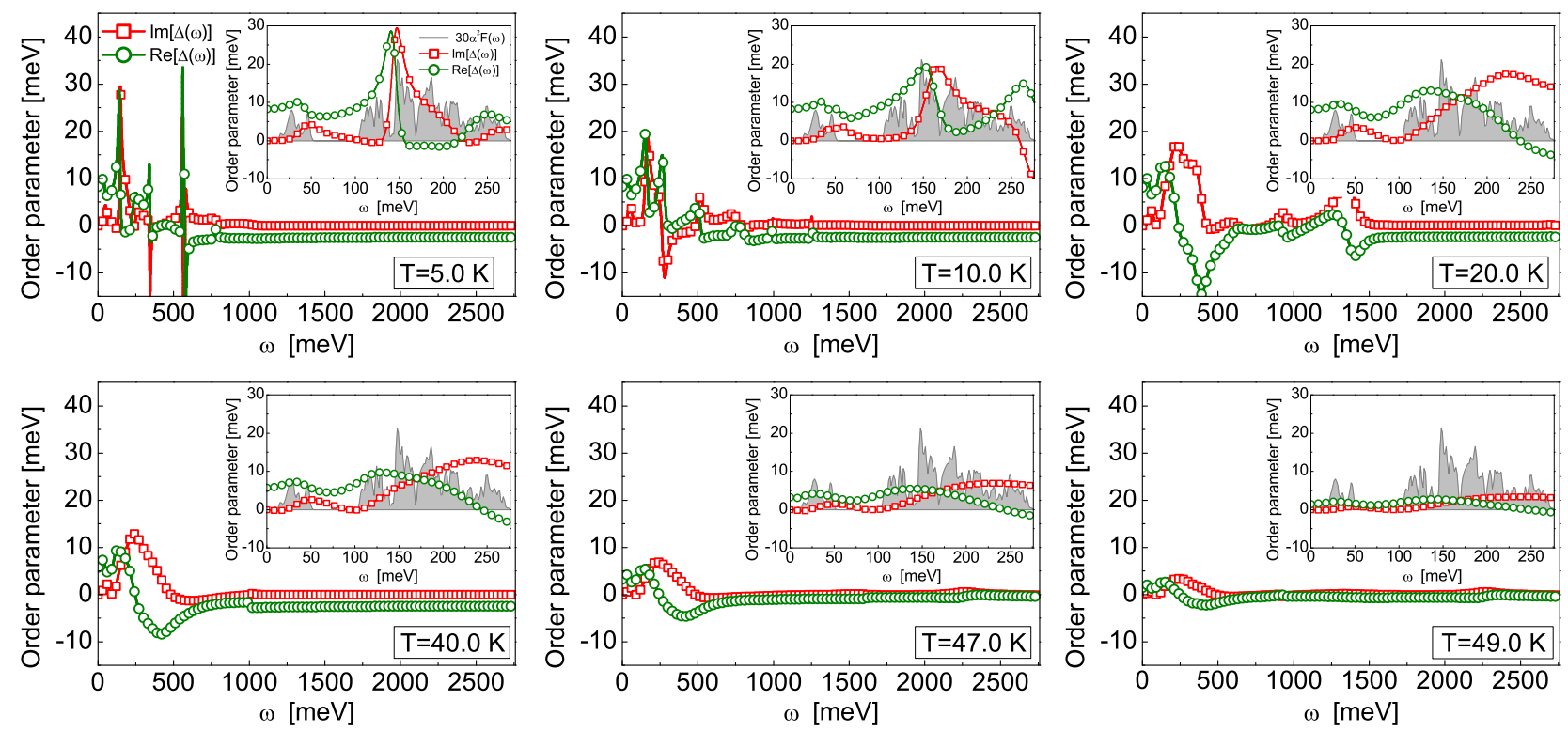

Fig. 5. The real and the imaginary parts of the order parameter on the real axis for selected values of the temperature and frequency range: $\omega \in\left\langle 0, \omega_{c}\right\rangle$. Additionally, the insets present the results for $\omega \in\left\langle 0, \Omega_{\max }\right\rangle$ on the background of rescaled Eliashberg function $\left(30 \alpha^{2} F(\omega)\right)$.

and

$$
\Delta U \equiv \int_{T_{\mathrm{C}}}^{T}\left[C^{S}(T)-C^{N}(T)\right] d T
$$

On this basis, we can conclude that the entropy and the internal energy of the superconducting state are lower than that of the normal state owing to the ordering of the electrons into pairs [36].

Based on the knowledge of the value of the critical temperature, the thermodynamic critical field and the specific heat of the superconducting and the normal state, it is possible to calculate the fundamental thermodynamic relations:

$$
R_{H} \equiv \frac{T_{C} C^{N}\left(T_{C}\right)}{H_{C}^{2}(0)} \quad \text { and } \quad R_{C} \equiv \frac{\Delta C\left(T_{C}\right)}{C^{N}\left(T_{C}\right)} .
$$

Due to the strong-coupling and retardation effects existing in studied system, the values determined for dimensionless ratios $R_{H}$ and $R_{C}$ differ from the predictions of the BCS theory (0.168 and 1.43 , respectively) $[37,38]$. In particular, for $\mathrm{NbH}_{4}$ at $300 \mathrm{GPa}$ we obtained $R_{H}=0.154$ and $R_{C}=2.12$. Above ratios play very important role in theory of superconductivity because they can be easily determined in experimental way and compared with theoretical predictions. However, in the case of $\mathrm{NbH}_{4}$ the experimental results are still lacking.

In order to calculate the third dimensionless ratio of the energy gap to the critical temperature $R_{\Delta} \equiv$ $2 \Delta(0) / k_{B} T_{C}$, the imaginary axis solution should be analytically continuing to the real frequencies.

In Figure 5, the shape of the order parameter for selected values of temperature and for the range of the frequencies from 0 to $\omega_{c}$ has been presented on the real axis. It can be observed, that for the low frequencies, the nonzero values are taken only by the real part of $\Delta(\omega)$. The imaginary part is linked to dissipative processes (absorption or emission of phonons). At zero temperature and zero frequency these processes are not possible and the gap function is real [39]. At non-zero temperature and low frequency they are very small, which explains the behaviour noticed in the insets of Figure 5. For the high frequency we can note that functions $\operatorname{Re}[\Delta(\omega)]$ and $\operatorname{Im}[\Delta(\omega)]$ are correlated with the shape of the Eliashberg function. Next, we can observe the characteristic sequence of the local maximums and minimums. Let us note, that because of the repulsive Coulomb pseudopotential, the real and imaginary part of the order parameter can give negative values. In the range of the frequencies higher than $1500 \mathrm{meV}$, the values of the order parameter becomes saturated and focuses around the value of zero.

Moreover, in Figure 6 the order parameter on the complex plane for the selected temperatures and for the frequencies range from 0 to $2 \Omega_{\max }$ has been plotted. We have found that the values of $\Delta(\omega)$ form the spirals with the radius that decreases together with the temperature growth. One can also see, that the effective electron-electron interaction is attractive $(\operatorname{Re}[\Delta(\omega)]>0)$ in the range of the frequencies from zero to $\sim 0.89 \Omega_{\max }$.

The physical value of the energy gap at the Fermi energy level has been calculated on the basis of the equation [40]:

$$
\Delta(T)=\operatorname{Re}[\Delta(\omega=\Delta(T), T)] .
$$

In Figure 7A, the full dependence of the energy gap at the Fermi level as a function of the temperature has been presented. The obtained result proves, that the maximum value of the energy gap becomes saturated for the temperature equal to $5 \mathrm{~K}$. For this reason we assumed $\Delta(0) \simeq \Delta(T(0))=8.35 \mathrm{meV}$. Next, the dimensionless 


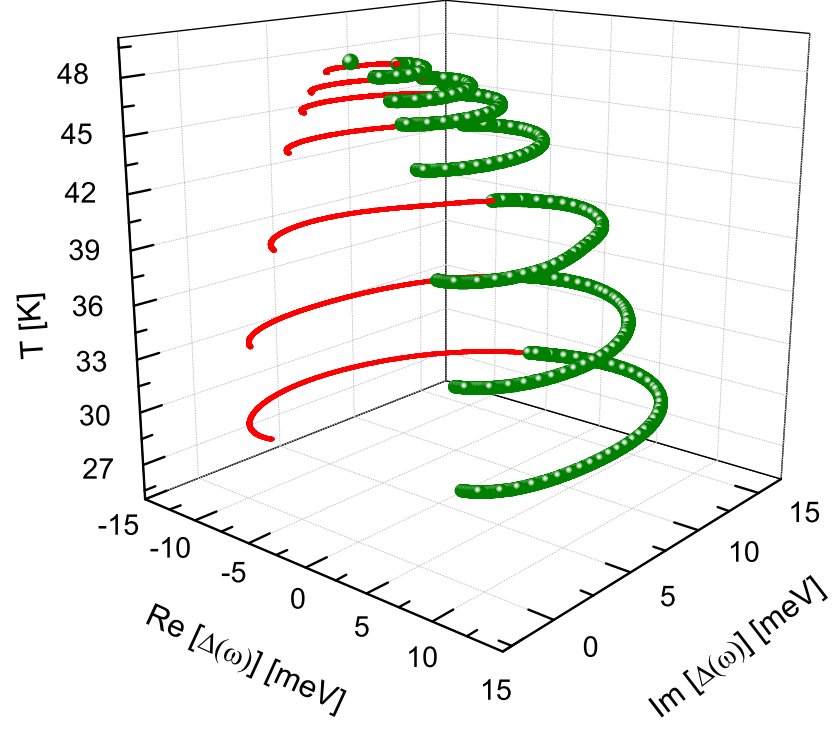

Fig. 6. The order parameter on the complex plane for the selected values of the temperature. The bold lines represent the solutions for $\omega \in\left\langle 0, \Omega_{\max }\right\rangle$; whereas regular lines correspond to the solutions for $\omega \in\left(\Omega_{\max }, 2 \Omega_{\max }\right\rangle$.

ratio $R_{\Delta}$ has been determined. For $\mathrm{NbH}_{4}$ at $300 \mathrm{GPa}$, we have obtained: $R_{\Delta}=3.91$. The above result is bigger than in the BCS model, where the dimensionless parameter $R_{\Delta}$ is equal to 3.53 [37]. It proved that the Eliashberg theory for strong-coupling $\mathrm{NbH}_{4}$ superconductor should be used instead of the BCS model. This is due to the fact that the thermodynamic properties of the compounds with $\lambda>0.5$ cannot be properly calculated within the framework of the weak-coupling theory [41]. It is worth emphasising that from the physical point of view, this is caused by the existence of the strong-coupling and retardation effects, which are omitted in the BCS theory. In the framework of the Eliashberg approach, these effects are characterized by the parameter $k_{B} T_{C} / \omega_{\text {ln }}$, where $\omega_{\text {ln }}$ denotes the logarithmic phonon frequency:

$$
\omega_{\mathrm{ln}} \equiv \exp \left[\frac{2}{\lambda} \int_{0}^{\Omega_{\max }} d \Omega \frac{\alpha^{2} F(\Omega)}{\Omega} \ln (\Omega)\right] .
$$

For the examined compound we have received $\omega_{\ln }=$ $76.59 \mathrm{meV}$. In the case of $\mathrm{NbH}_{4}$ at $300 \mathrm{GPa}$, we have obtained: $k_{B} T_{C} / \omega_{\text {ln }} \simeq 0.056$, when in the weak-coupling limit, one can assume zero.

In the last step, the value of the electron effective mass $\left(m_{e}^{\star}\right)$ has been determined on the basis of the real part of the wave function renormalization factor:

$$
m_{e}^{\star}=\operatorname{Re}[Z(0)] m_{e},
$$

where $m_{e}$ is the electron band mass.

In Figure 7B the functions $\operatorname{Re}[Z(\omega)]$ and $\operatorname{Im}[Z(\omega)]$ for $T_{C}$ and $\omega \in\left\langle 0, \Omega_{\max }\right\rangle$ have been presented. Moreover, the full dependence of $Z(\omega)$ as a function of the temperature has been presented in Figure $7 \mathrm{C}$. The obtained results prove, that the maximum value of $Z(\omega)$ is observed for
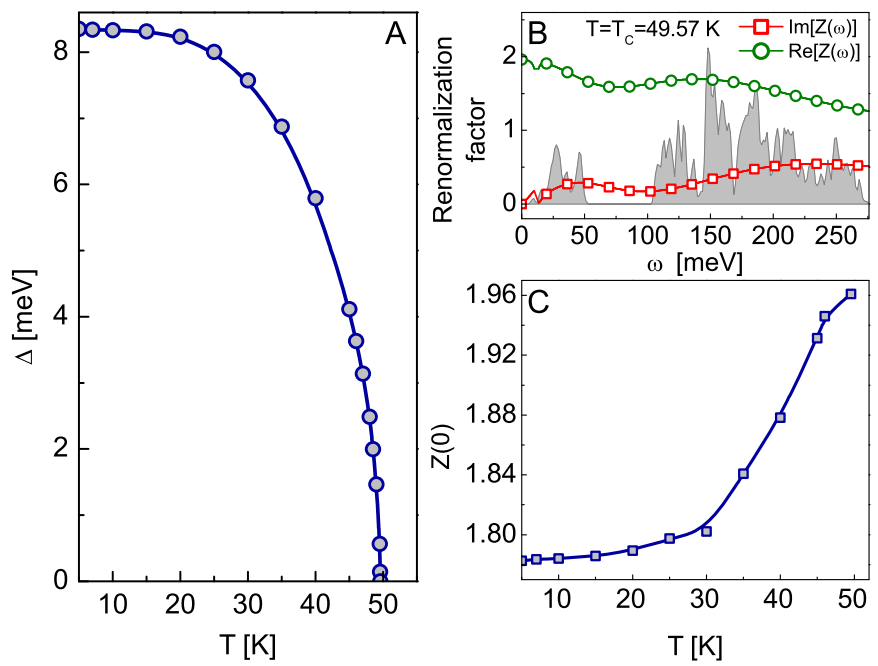

Fig. 7. (A) The full dependence of the order parameter on the temperature. (B) The dependence of the real and imaginary part of the wave function renormalization factor on the frequency for the critical temperature (in the background the rescaled Eliashberg function $\left(3 \alpha^{2} F(\omega)\right)$ has been plotted). (C) The full dependence of the wave function renormalization factor on the temperature.

the critical temperature. For this reason we assumed that the effective mass $m_{e}^{\star}$ is high in the whole range of the considered temperatures, and its highest value is equal to $1.96 m_{e}$ for $T=T_{C}$.

\section{Conclusions}

In the present paper, we have studied the superconducting state of the $\mathrm{NbH}_{4}$ compound at $300 \mathrm{GPa}$. The high value of electron-phonon coupling constant $(\lambda=0.82)$ indicating the necessity of using the Eliashberg equations to determine the thermodynamic properties of this material. In the first step, it has been stated that the critical temperature is high for considered value of the Coulomb pseudopotential $\left(T_{C}=49.57 \mathrm{~K}\right.$ for $\left.\mu^{\star}=0.1\right)$. Then, the order parameter, the specific heat and the thermodynamic critical field have been determined. On the basis of these results, it has been proven that the values of the dimensionless ratios $R_{\Delta}, R_{C}$ and $R_{H}$ differ significantly from the predictions of the BCS theory. In particular, we obtained the following results $3.91,2.12,0.154$, respectively. It is connected with fact that the Eliashberg formalism in contrast to BCS model does not omit the strong-coupling and retardation effects. The electron effective mass has been also calculated. The maximum value of $m_{e}^{\star}$ is equal to $1.96 m_{e}$ for critical temperature.

The authors wish to thank Prof. K. Dziliński for providing excellent working conditions and the financial support. All numerical calculations have been based on the Eliashberg function sent to us by Ph.D. Guoying Gao to whom we are very 
thankful. Additionally, we are grateful to the Czȩstochowa University of Technology - MSK CzestMAN for granting access to the computing infrastructure built in the project No. POIG.02.03.00-00-028/08 "PLATON - Science Services Platform".

\section{References}

1. N.W. Ashcroft, Phys. Rev. Lett. 21, 1748 (1968)

2. N.W. Ashcroft, Phys. Rev. Lett. 92, 187002 (2004)

3. D.C. Lonie, J. Hooper, B. Altintas, E. Zurek, Phys. Rev. B 87, 054107 (2013)

4. J. Hooper, B. Altintas, A. Shamp, E. Zurek, J. Phys. Chem. C 117, 2982 (2013)

5. J. Hooper, T. Terpstra, A. Shamp, E. Zurek, J. Phys. Chem. C 118, 6433 (2014)

6. M.I. Eremets, I.A. Trojan, S.A. Medvedev, J.S. Tse, Y. Yao, Science 319, 1506 (2008)

7. X.J. Chen, V.V. Struzhkin, Y. Song, A.F. Goncharov, M. Ahart, Z. Liu, H. Mao, R.J. Hemley, Proc. Natl. Acad. Sci. USA 105, 20 (2008)

8. D.Y. Kim, R.H. Scheicher, S. Lebégue, J. Prasongkit, B. Arnaud, M. Alouani, R. Ahuja, Proc. Natl. Acad. Sci. USA 105, 16454 (2008)

9. O. Degtyareva, J.E. Proctor, C.L. Guillaume, E. Gregoryanz, M. Hanfland, Solid State Commun. 149, 1583 (2009)

10. M. Hanfland, J.E. Proctor, Ch.L. Guillaume, O. Degtyareva, E. Gregoryanz, Phys. Rev. Lett. 106, 095503 (2011)

11. D.Y. Kim, R.H. Scheicher, Ch.J. Pickard, R.J. Needs, R. Ahuja, Phys. Rev. Lett. 107, 117002 (2011)

12. X.-F. Zhou, A.R. Oganov, X. Dong, L. Zhang, Y. Tian, H.-T. Wang, Phys. Rev. B 84, 054543 (2011)

13. X. Jin, X. Meng, Z. He, Y. Ma, B. Liu, T. Cui, G. Zou, H. Mao, Proc. Natl. Acad. Sci. USA 107, 9969 (2010)

14. Y. Li, G. Gao, Y. Xie, Y. Ma, T. Cui, G. Zou, Proc. Natl. Acad. Sci. USA 107, 15708 (2010)

15. G. Zhong, Ch. Zhang, X. Chen, Y. Li, R. Zhang, H. Lin, J. Phys. Chem. C 116, 5225 (2012)

16. G. Gao, H. Wang, A. Bergara, Y. Li, G. Liu, Y. Ma, Phys. Rev. B 84, 064118 (2011)

17. R. Szczesśniak, A.P. Durajski, Supercond. Sci. Technol. 27, $015003(2014)$

18. H. Wang, J.S. Tse, K. Tanaka, T. Iitaka, Y. Ma, Proc. Natl. Acad. Sci. USA 109, 6463 (2012)

19. R. Szczȩśniak, A.P. Durajski, Solid State Sci. 25, 45 (2013)
20. H. Fujihisa, Y. Nakamoto, M. Sakata, K. Shimizu, T. Matsuoka, Y. Ohishi, H. Yamawaki, S. Takeya, Y. Gotoh, Phys. Rev. Lett. 110, 235501 (2013)

21. G. Gao, R. Hoffmann, N.W. Ashcroft, H. Liu, A. Bergara, Y. Ma, Phys. Rev. B 88, 184104 (2013)

22. G.M. Eliashberg, Soviet. Phys. J. Exp. Theor. Phys. 11, 696 (1960)

23. M. Lüders, M.A.L. Marques, N.N. Lathiotakis, A. Floris, G. Profeta, L. Fast, A. Continenza, S. Massidda, E.K.U. Gross, Phys. Rev. B 72, 024545 (2005)

24. R. Szczȩśniak, Acta Phys. Pol. A 109, 179 (2006)

25. R. Szczȩśniak, D. Szczȩśniak, K.M. Huras, Phys. Stat. Sol. B 251, 178 (2014)

26. R. Szczȩśniak, D. Szczȩśniak, Phys. Stat. Sol. B 249, 2194 (2012)

27. A.P. Durajski, R. Szczȩśniak, M.W. Jarosik, Phase Transit. 85, 727 (2012)

28. R. Szczȩśniak, M.W. Jarosik, D. Szczȩśniak, Physica B 405, 4897 (2010)

29. R. Szczȩśniak, D. Szczȩśniak, Solid State Commun. 152, $779(2012)$

30. A.P. Durajski, R. Szczesśniak, Acta Phys. Pol. A 126, 342 (2014)

31. R. Szczȩśniak, Solid State Commun. 145, 137 (2008)

32. J.P. Carbotte, Rev. Mod. Phys. 621027 (1990)

33. W.L. McMillan, Phys. Rev. 167, 331 (1968)

34. P.B. Allen, R.C. Dynes, Phys. Rev. B 12, 905 (1975)

35. Y. Wang, T. Plackowski, A. Junod, Physica C 355, 179 (2001)

36. J. Sólyom, in Fundamentals of the Physics of Solids: Normal, Broken-Symmetry, and Correlated Systems (Springer, 2011), Vol. 3

37. J. Bardeen, L.N. Cooper, J.R. Schrieffer, Phys. Rev. 106, $162(1957)$

38. J. Bardeen, L.N. Cooper, J.R. Schrieffer, Phys. Rev. 108, 1175 (1957)

39. R. Combescot, Phys. Rev. B 51, 11625 (1995)

40. E. Cappelluti, C. Grimaldi, L. Pietronero, S. Strässler, G.A. Ummarino, Eur. Phys. J. B 21, 383 (2001)

41. J. Bauer, J.E. Han, O. Gunnarsson, Phys. Rev. B 84, 184531 (2011)

Open Access This is an open access article distributed under the terms of the Creative Commons Attribution License (http://creativecommons.org/licenses/by/4.0), which permits unrestricted use, distribution, and reproduction in any medium, provided the original work is properly cited. 